\title{
Arthroscopic Surgery of the Elbow; Indications, Contra-Indications, Complications and Operative Technique
}

\author{
Frank Theodoor Gabriel Rahusen ${ }^{1}$, Denise Eygendaal ${ }^{2}$ \\ ${ }^{1}$ St. Jansgasthuis Weert, Weert, Netherlands \\ ${ }^{2}$ Amphia Ziekenhuis Breda, Breda, Netherlands \\ E-mail: fthgrahusen@hetnet.nl, denise@eygendaal.nl \\ Received February 8, 2011; revised April 7, 2011; accepted June 2, 2011
}

\begin{abstract}
Arthroscopy of the elbow was first described by Burman in 1931. In this first article about arthroscopy of the elbow in the journal of bone and joint surgery, he concluded that the elbow joint was not suitable for arthroscopy; the joint was too small and the neurovascular structures in the anterior compartment of the elbow were close. In 1932 he revised his original article with some technical modifications and slowly arthroscopy of the elbow was performed more often. In the late 1980's arthroscopic surgery of the elbow became more and more popular. In this article an overview is given of the indications for elbow arthroscopy, the surgical technique is described in detail and the possible complications are highlighted.
\end{abstract}

Keywords: Elbow, Arthroscopy, Surgical Technique, Overview

\section{Introduction}

Since the eighties, arthroscopy of the elbow joint is performed more often as the understanding of the arthroscopic anatomy and its disorders have become clearer.

Small performed an epidemiologic survey and in the late 1980 's; only $0.77 \%$ of all arthroscopies in those days performed were arthroscopies of the elbow [1].

Currently common indications for arthroscopy of the elbow are symptomatic loose bodies, posterior impingement, osteochondritis dissecans (OCD), long standing lateral epicondylitis, persistent synovitis which require debridement or diagnostic biopsy, and stiff elbow due to arthritic changes or due to posttraumatic deformity [2]. Peri-articular, endoscopic, techniques as a bursectomy, a tenoscopy of the biceps tendon or decompression of the ulnar nerve will not be discussed in this overview.

Since the popularity of sports, especially the overhead throwing and racket sports, is growing, the incidence of elbow pathology is increasing.

The technique of arthroscopic surgery has been improved dramatically last ten years; the incidence of complication as neurovascular damage is acceptable.

In this overview the indications for elbow arthroscopy are listed, the surgical technique is described in detail and the possible complications are highlighted.

The most common complication in elbow arthroscopy is neurologic deficit post-operatively. Also post-operative elbow stiffness, persistent portal drainage and infection have been mentioned. O'Driscoll and Morrey showed an overall $10 \%$ risk in their review of 70 patients [3]. Other papers show complication rates between $0 \%$ and $15 \%$. There are several techniques to perform an elbow arthroscopy. Mainly there are 3 different patient positions; supine, prone and lateral decubitus [4]. All have their own benefits in different indications.

\section{Indications for Elbow Arthroscopy}

\subsection{Diagnostic Elbow Arthroscopy}

Diagnostic elbow arthroscopy is not advocated, but may be helpful when the clinical diagnosis is unclear. Also undetected elbow instability in overhead athletes can be seen during diagnostic elbow arthroscopy. Timmermans, et al. described an arthroscopic valgus instability test, in which a valgus load is applied to the elbow in 70 degrees of flexion during the arthroscopy. The medial comartment opens up and can be inspected. If the medial compartment open up for more than 2 - $3 \mathrm{~mm}$, Field and 
Altchek concluded that the ulnar collateral ligament can be torn [5].

\subsection{Loose Bodies}

Symptomatic loose bodies are the most common indication for arthroscopy of the elbow. Loose bodies are often a symptom of an underlying disorder which has to be assessed and treated. Often, loose bodies are the result of a trauma, resulting in osteochondral fractures or fracture of (asymptomatic) osteofytes. Loose bodies are also formed in longstanding OCD or in synovial chondromatosis as described by Flury, et al.; in both diseases additional arthroscopic treatment is indicated [6].

Loose bodies may hide in any part of the elbow joint. Most commonly they are hided posteriorly in the olecranon fossa, at the posterior aspect of the radial capitellar articulation or anteriorly in the coronoid fossa. In case of loose bodies the surgeon therefore should assess all compartments of the elbow joint during the arthroscopic procedure. Preoperative radiographs and computer tomography is indicated and delineate in most cases the location of loose bodies.

\subsection{Posterior Impingement}

Posterior impingement of the elbow is an uncommon disorder in the general young population; it is usually seen in patients that overuse their elbow during specific sporting activities as overhead throwing or tennis. During the throwing motion, in baseball, for example, the elbow moves during late cocking and acceleration phases from $110^{\circ}$ to $20^{\circ}$ of flexion with velocities up to $3000 \mathrm{deg} / \mathrm{sec}$. This combination of valgus forces and rapid extension results in tensile forces along the medial side, compression on the lateral portion of the elbow, and shear forces in the posterior compartment. This combination is called valgus extension overload syndrome and forms the basic pathologic model behind posterior impingement of the elbow as formation of bony or soft tissue in the posterior compartment results in mechanical abutment leading to complaints of the posterior compartment during extension. The exact fit of the olecranon in the olecranon fossa of the humerus is critical for a maximal extension and, therefore, for the function of the elbow. In particular the maximal extension needed in most overhead sports is reduced, leading to complaints of the posterior compartment of the elbow. The athlete complains of pain posteriorly at the elbow, joint effusion, locking, crepitus, and a decrease in range of motion, most notably an extension deficit. X-rays, especially an axial view, may be helpful to detect osteophytes on the olecranon or on the borders of the posterior fossa. More sensitive is an
MRI with intra-articular contrast; sensitivity for posterior soft tissue or loose bodies is nearly $90 \%$. If conservative treatment of posterior impingement is not successful; arthroscopy of the elbow can be successfully used in these patients as described in an earlier review of Rahusen, et al. [7].

\subsection{Osteochondritis Dissecans}

Osteochondritis dissecans is a localized condition involveing the articular surface that results in the separation of a segment of articular cartilage and subchondral bone. The most common site of osteochondritis dissecans of the elbow is the capitellum although lesions have been reported in the trochlea, radial head, as well as the olecranon and olecranon fossa. Osteochondritis dissecans generally occurs in athlete's ages 11 to 21 years who report a history of overuse. The osteonecrotic lesion involves only a segment of capitellum, located primarily at a central or anterolateral position. Appropriate treatment of this disorder remains controversial.

Often treated with benign neglect, this condition is a potentially sport-ending injury for anathlete, with longterm sequelae of degenerative arthritis. The surgical option is fragment excision with debridement of the necrotic lesion.

\subsection{Longstanding Lateral Epicondylitis}

Lateral epicondylitis or tennis elbow is a common disorder in primary care. It is rather related to manually intensive work, requiring forceful and repetitive rotation of the forearm, wrist extension or flexion (e.g. in mechanics, butchers, construction workers)The incidence of lateral humeral epicondylitis in general practice is estimated at 4 - 7 per 1000 patients per year, with a peak between 35 and 54 years of age. Lateral epicondylitis is generally a self-limiting condition. The average duration of a typical episode varies from six months to two years, but most patients (90\%) respond to conservative treatments and recover within one year. Surgical treatment is recommended for those patients who are not responding to conservative treatment after at least six months to a year. Surgical techniques are various, including open, percutaneous and arthroscopic treatment. Research investigating which approach is superior, reveals that the less invasive approaches (percutaneous or arthroscopic) allow faster return to work, than the open procedure. Arthroscopic release is potential beneficiary because an arthroscopic evaluation of the whole joint can be done during the procedure. Also other intra-articular problems, which have been described in up to $50 \%$ of all cases, can be addressed simultaneously. Baker, et al. showed a return 
to work at an average of 2.2 weeks and a grip strength of $96 \%$ compared to the unaffected limb [8].

\subsection{Persistent Synovitis}

Persistent synovitis of the elbow, due to rheumatoid arthritis or other inflammatory pathology, which is not responding to conservative treatment, can be indication for debridement or diagnostic biopsy in cases the cause of the synovitis is unknown. De boer, et al. showed this in their studies [9].

Septic arthritis of the elbow can be treated with arthroscopy as well.

\subsection{Stiff Elbow}

Loss of motion is a common complication in degeneration or after elbow trauma. Restoration of joint motion especially in the posttraumatic stiff elbow can be a difficult, time-consuming, and a costly challenging [10]. Elbow contractures can be the result of intrinsic (intraarticular) or extrinsic (extra-articular) causes [11-13]. In most posttraumatic contractures both intrinsic and extrinsic causes play a role. Established contractures should be treated initially with physical therapy and staticprogressive splinting. Patients who have failed a minimum of 6 to 12 months of non-surgical management and who are motivated to comply with a strict postoperative rehabilitation program are candidates for surgical release [14].

Arthroscopic arthrolysis of stiff elbows has been introduced as a safe, but technical demanding technique [15]. The indications for surgery depend on the patient's functional needs. Morrey, et al. stated that an elbow needs a minimal range of motion (ROM) of 100 degrees flexion /extension and 100 degrees of pronation/supination to function adequately in daily life [16]. However in specific groups of patients, as professional athletes, even a slight extension deficit of 20 degrees can result in a dysfunction of the elbow.

Disadvantages of elbow arthroscopy include the inability to deal with ulnar nerve disease or heterotopic ossification and the length of the procedure.

\subsection{Surgical Technique}

Arthroscopy of the elbow is routinely done under general anesthesia without additional regional anesthesia, to allow postoperative evaluation of the integrity of the nerves. With the patient still in supine position, the elbow is examined for range of motion and for instability. Then the patient is placed in supine or prone position. We generally perform the arthroscopy with the patient in a lateral decubitus position with the upper arm in a support with tourniquet. In this position all compartments are easily accessible. Alternatively the patient is positioned in prone, with the arm hanging down, or supine with the arm suspended and the elbow passively flexed in $90^{\circ}$.

It is very important to identify and mark the bony landmarks and the ulnar nerve before insufflating the joint with saline. After this the joint is filled with $30 \mathrm{ml}$ of saline, before making the first portal. Backflow of fluid verifies proper placement. Cadaveric studies have demonstrated that joint insufflation significantly increases the distance between the joint surfaces and neurovascular structures, thus helping to protect them from injury during joint entry and during the use of intraarticular instrumentation.

When the arthroscopic sheath is inserted only blunt trocarts should be used. When creating portals, the surgeon should avoid penetrating the subcutaneous tissue, thereby helping to prevent injury to the superficial cutaneous nerves. A mosquito clamp can be used to spread tissues down to the capsule.

A high pump pressure during the arthroscopy can re sult in loss of fluid in the soft tissues, resulting in compartment syndrome of the fore-arm. Insufflation of the joint above a pressure of more than $50 \mathrm{mmHg}$ should be avoided.

Portal placement is at the surgeon's distinction. Many portals have been described and specific portals have their own benefits. Usually the direct-lateral, anteromedial, anterolateral and the proximal-medial portals are used for the anterior, lateral and medial compartment. The straight-posterior and posterolateral portals are used for the arthroscopy of the posterior compartment.

The initial survey of the anterior aspect of the elbow is performed using the proximal medial portal: This allows localization of the loose fragments, their approximate position, and it allows the assessment of the medial and lateral gutters. The loose body is noted, and the proximal anterolateral portal is established by using the spinal needle to ensure adequate access with the pending entry. This portal is established, and the loose body is located, grasped, and removed. In many cases, it may be useful to "pin" the loose fragment with a spinal needle to provide resistance for grasping the loose piece of bone. Additionally, the portal may need to be enlarged to allow full excision of the fragment, or alternatively, it may be removed piece by piece. Using the antero-lateral portal the tip of the coronoid can be debrided, as well as the coronoid fossa.

Most OCD are not visible from anterior. Lateral epicondylitis is debrided in the manner as well. With a standard arthroscopy of the posterior compartment of the 
elbow the olecranon fossa is cleared of soft tissue. Osteofytes at the posteromedial site of the proximal ulna or distal humerus can be debrided. Using a mid posterior portal and a postero-lateral portal the posterior compartment can be debrided using a $5.5 \mathrm{MM}$ oscillating shaver and a 4 MM cylindric shaver burr.

In case of arthrocopic artrholysis all osteofytes, loose bodies and fibrotic tissue are removed. The anterior compartment is examined using an antero-medial portal after a careful palpation of the ulnar nerve and intramuscular septum; a second portal antero lateral is created outsidein as described previously. With a 5.5 MM oscillating shaver a synovectomie is performed. With a $4 \mathrm{MM}$ cylindric shaver burr, the coronoid process and the coronoid fossa are debrided. In the end the anterior capsule is released using a punch from medial to lateral. After treatment consists of Continuous Passive Motion device (CPM) for the first 24 hours, continuously, followed by a standardized program under supervision of a physiotherapist. The results of arthroscopic treatment compare favourably with those of open techniques with low rate of complications in both techniques. Elbow arthroscopy offers improved joint visualisation, reduced pain, smaller scars, accelerated rehabilitation and shorter hospital stay, potentially making arthroscopic release an outpatient procedure.

\subsection{Complications in Arthroscopy of the Elbow}

Most often complications of arthroscopy of the elbow are of neurologic origin. Usually the neurologic complications are transient but several authors describe total transection of the nerve, in particular the ulnar nerve. Transient radial nerve en median nerve problems have been reported, but the incidence is very low. Transient nerve deficiencies can be due to neuropraxia by compression of the nerve by instruments or by positioning of the patient on the table. Transection of the nerve mostly occurs by introduction of the scope or by capsulectomy anterolateral (radial nerve) or posteromedial (ulnar nerve) [17].

Excessive drainage from the portal sites has been described however the imported infection rate is low $[8,18]$. Temporary loss of motion is seen in most cases, but usually resolves within 6 - 8 weeks [8]

\section{Conclusions}

Indications for arthroscopy of the elbow are symptomatic loose bodies, posterior impingement, osteochondritis dissecans (OCD), long standing lateral epicondylitis, persistent synovitis which require debridement or diagnostic biopsy, and stiff elbow due to arthritic changes or due to posttraumatic deformity
The technique of arthroscopic surgery has been improved dramatically last ten years; the incidence of complication as neurovascular damage is acceptable.

\section{References}

[1] M. S. Burman, “Arthroscopy or the Direct Visualization of Joints: An Experimental Cadaveric Study,” Journal of Bone and Joint Surgery, Vol. 13, No. 4, 1931, pp. 669-695.

[2] N. C. Small, "Complications in Arthroscopy: The Knee and other Joints,” Arthroscopy, Vol. 2, No. 4, 1986, pp. 253-258. doi:10.1016/S0749-8063(86)80080-9

[3] S. W. O’Driscoll and B. F. Morrey, “Arthroscopy of the Elbow. Diagnostic and Therapeutic Benefits and Hazards,” Journal of Bone \& Joint Surgery, Vol. 74, No. 1, 1992, pp. 84-94.

[4] L. A. Timmermans, "Histology and Arthroscopic Anatomy of the Ulnar Collateral Ligament of the Elbow," American Journal of Sports Medicine, Vol. 22, No. 5, 1994, pp. 667-673. doi:10.1177/036354659402200515

[5] L. A. Field and D. W. Altchek, "Evaluation of the Arthroscopic Valgus Instability Test of the Elbow," American Journal of Sports Medicine, Vol. 24, No. 2, 1996, pp. 177-181. doi:10.1177/036354659602400210

[6] M. P. Flury, J. Goldhahn, S. Drerup and B. R. Simmen, "Arthroscopic and Open Options for Surgical Treatment of Chondromatosis of the Elbow," Arthroscopy, Vol. 24, No. 5, 2008, pp. 520-525. doi:10.1016/j.arthro.2007.12.002

[7] F. T. Rahusen, J. M. Brinkman and D. Eygendaal, "Arthroscopic Treatment of Posterior Impingement of the Elbow in Athletes: A Medium-Term Follow-Up in Sixteen Cases," Journal of Shoulder and Elbow Surgery, Vol. 18, No. 2, 2009, pp. 279-282. doi:10.1016/j.jse.2008.10.015

[8] C. L. Baker, “Arthroscopy of the Elbow,” American Journal of Sports Medicine, Vol. 27, No. 2, 1999, pp. 251-264.

[9] Y. A. De Boer, C. H. Van den Ende, D. Eygendaal, I. M. Jolie, J. M. Hazes and P. M. Rozing, "Clinical Reliability and Validity of the Elbow Functional Assessment in Rheumatoid Arthritis,” Journal of Rheumatology, Vol. 26, No. 9, 1999, pp. 1909-1917.

[10] A. L. Lindenhovius and J. B. Jupiter, "The Posttraumatic Stiff Elbow: A Review of the Literature," Journal of Hand Surgery, Vol. 32, No. 10, 2007, pp. 1605-1623. doi:10.1016/j.jhsa.2007.09.015

[11] C. M. Ball, M. Meunier, L. M. Galatz, R. Calfee and K. Yamaguchi, "Arthroscopic Treatment of Post-Traumatic Elbow Contracture," Journal of Shoulder and Elbow Surgery, Vol. 11, No. 6, 2002, pp. 624-629. doi:10.1067/mse.2002.126770

[12] J. W. T. Byrd, "Elbow Arthroscopy for Arthrofibrosis after Type I Radial Head Fractures," Arthroscopy, Vol. 10, No. 2, 1994, pp. 162-165. doi:10.1016/S0749-8063(05)80087-8

[13] M. S. Cohen, "Hastings H 2nd. Post-Traumatic Contracture of the Elbow: Operative Release Using a Lateral Col- 
lateral Ligament Sparing Approach,” Journal of Bone and Joint Surgery, Vol. 80, 1998, pp. 805-812.

doi:10.1302/0301-620X.80B5.8528

[14] U. Gundlach and D. Eygendaal, "Surgical Treatment of Posttraumatic Stiffness of the Elbow, 2-Year Outcome in 21 Patients after Column Procedure,” Acta orthopaedica, Vol. 79, No. 1, 2008, pp. 74-77. doi:10.1080/17453670710014798

[15] D. Sahajpal, T. Choi and T. W. Wright, "Arthroscopic Release of the Stiff Elbow," Journal of Hand Surgery, Vol. 34, No. 3, 2009, pp. 540-544.

doi:10.1016/j.jhsa.2008.12.018
[16] B. F. Morrey, "Functional Anatomy of the Ligaments of the Elbow," Clinical Orthopaedics and Related Research, Vol. 201, 1985, pp. 84-90.

[17] T. Haapaniemi, M. Berggeren and L. Adolfsson, "Case Report Complete Transection of the Median and Radial Nerves during Arthroscopic Release of Post-Traumatic Elbow Contracture,” Arthroscopy, Vol. 15, No. 7, 1999, pp. 784-787. doi:10.1016/S0749-8063(99)70015-0

[18] E. W. Kelly, B. F. Morrey and S. W. O’Driscoll, "Complications of Elbow Arthroscopy," Journal of Bone and Joint Surgery, Vol. 83, 2001, pp. 25-34. 